\title{
ISOLECTINS OF PHYTOHEMAGGLUTININ ARE ABLE TO INDUCE APOPTOSIS IN HEp-2 CARCINOMA CELLS IN VITRO
}

\author{
T.O. Kochubei*, O.V. Maksymchuk, O.O. Piven, L.L. Lukash \\ Institute of Molecular Biology and Genetics, NAS of Ukraine, Kyiv 03680, Ukraine
}

\begin{abstract}
Aim: To study the effects of total phytohemagglutinin (PHA) and its isolectins on cell death and apoptosis in human HEp-2 carcinoma cells and to analyze the possible molecular mechanisms of lectin induced apoptosis. Materials and Methods: The commercial preparation of the kidney beans (Phaseolus vulgaris) lectins and HEp-2 cells were used. Apoptosis index was determined using acridine orange and ethidium bromide staining. The expression levels of apoptosis mediator cleaved caspase-3 and proapoptotic Bax protein were studied by Western blot analysis. The gene expression levels were analyzed by qPCR. Results: PHA and its isolectins induced apoptosis in HEp-2 cells accompanied by the increased expression of caspase-3 cleaved form, with PHA-E being the most effective. The treatment of HEp-2 cells with PHA or its isolectins resulted in a marked increase of Bax on both mRNA and protein levels. Conclusions: PHA and its isolectins were shown to induce the apoptosis in human HEp-2 carcinoma cells via increasing proapoptotic protein Bax and activating caspases-3.
\end{abstract}

Key Words: phytohemagglutinin, isolectins, apoptosis, caspase-3, Bax.

Phytohemagglutinin (PHA) is lectin of Phaseolus vulgaris and it is one of the most common and widely studied lectin. Mitogenic activity of PHA is known since $60^{\text {th }}$ of the XX century. It's important to note that this lectin has an original molecular structure, namely PHA is a mixture of different isolectins, including erythroagglutinin (PHA-E) and leukoagglutinin (PHA-L). Each of these isolectins is tetramer, which consists of $\mathrm{E}$ - and L-subunits [1].

As it has been mentioned, PHA is widely studied protein used in many cytological studies [2]. It should be noted that, beside its mitogenic properties, PHA has some other biological activities. It is known that PHA accelerates growth of Allium cepa roots and Rh. coccineus shoots on the first stages of their growth [1]. Also the toxicity of PHA was observed in the animal models. It has been revealed that PHA is toxic for larvae of some beetles that eat seeds of cereals and legumes [3]. The toxic effect of PHA lectins was also analysed in the rodent models. The toxicity of PHA lectins for rats has not been observed but the growth delay of animals and reduction of nitrogen fixation in the cells of mucous membrane of the intestine was registered [1]. The author believe that lectins of $P h$. vulgaris can interact with the glycocalyx of enterocytes and, as a result, cause the cell damage. Additionally, the PHA was shown to affect the lipid catabolism through lowering the level of insulin in the blood plasma [4] and strongly inhibiting the hydrochloric acid secretion by basal cells in the rat stomach [5]. Recently the capability of PHA to induce food allergies has been reported [6].

It should be noted that, except of demonstration its mitogenic and cytotoxic activity, the PHA can also cause the apoptosis. As it was reported earlier, PHA-E is able to induce apoptosis in human lung cancer cells through mitochondrial pathway [7]. In our previous

Submitted: August 13, 2014

*Correspondence: E-mail: kochubei2009@ukr.net Abbreviations used: PHA - phytohemagglutinin; PHA-E - erythroagglutinin; PHA-L - leukoagglutinin. work we have also observed that of PHA and its isolectins affect cell proliferation and apoptosis. All studied proteins inhibited human cancer cell proliferation and survival in dose dependent manner [8]. We supposed that PHA through specific carb binding properties and its isolectins specifically can induce not only cells proliferation but also apoptosis. It gives consideration to this group of proteins as interesting and perspective for targeted anticancer therapies. In this line we would like to note that other group of scientists has found that PHA-L is able to recognize $\beta-1-6-\mathrm{N}$-acetylglucosamine residues of $\mathrm{N}$-glycans, increased expression of which is known to serve as a marker of cancer progression and metastasis [9]. However, proapoptotic mechanisms of PHA and its isolectins activity still remain unclear and should be elucidated. In this study we analysed the influence of PHA and its isolectins on apoptosis in human cancer cells and clarified possible signalling pathways endorsed by lectins activity.

\section{MATERIALS AND METHODS}

In our study we have analyzed the influence of the commercial preparation of the kidney beans (Ph. vulgaris) lectins: including PHA, PHA-E and PHA-L (Lectynotest, Lviv, Ukraine) on apoptosis in human cell culture. We have used the human HEp-2 carcinoma cells as a test system. The cells have been cultured according to standard method as described previously [10].

Influence of lectins (concentrations of each lectin were $1 ; 10$ and $100 \mu \mathrm{g} / \mathrm{ml}$, the treatment of cells lasted 4 hours) on the frequency of alive, dead and early apoptotic cells has been analyzed using acridine orange and ethidium bromide staining [11]. Microscopic analysis was performed using ML-2 fluorescent microscope (LOMO, Russian Federation).

Molecular mechanisms of apoptosis were investigated under the action of studied lectins at $1 \mu \mathrm{g} /$ $\mathrm{ml}$. The changes of effector caspase-3 (cleaved form) and Bax levels have been inspected by modified western blot analysis [12]. Total protein has been 
isolated [13] and separated by electrophoresis in 15\% polyacrylamide gel with $0.1 \%$ sodium dodecyl sulphate [14]. For evaluation of proteins expression levels the anti-cleaved caspase- 3 p11 polyclonal antibody (h 176)-R: sc-22171 (Santa Cruz Biotechnology, Inc., Santa Cruz, CA, USA) and anti-Bax polyclonal antibody (HPA027878, Sigma-Aldrich, Saint Louis, MO, USA) were used. For normalization of signals the blotting with anti-glyceraldehyde phosphate dehydrogenase (GAPDH) antibodies was perfomed. Results of western blot were visualized and calculated by ChemiDoc XRS+System with Image Lab Software (Bio-Rad, Hercules, CA, USA). The relative protein expression level is presented as a ratio of protein of interest to GAPDH signal on western blotting. All experiments have been carried out in triplets.

The total RNA has been isolated from cells using UltraClean Tissue \& Cells RNA Isolation Kit (Mo Bio Laboratories, Carlsbad, CA, USA) according to the manufacturer's protocol. cDNA has been synthesized by First Strand cDNA Synthesis Kit (Fermentas, Vilnius, Lithuania) by DNAse I treatment step, and qPCR has been performed with Maxima SYBR Green/Fluorescein qPCR Master Mix (Fermentas, Vilnius, Lithuania). All real-time reactions were done in an iCycler with MyIQ Single Color Real-Time PCR Detection System IQ5 software (Bio-Rad, Hercules, CA, USA). Then gene expression was presented by the $2^{-\triangle \Delta C T}$ value normalized to the reference gene for $\beta$-actin. The $\Delta \Delta \mathrm{C}_{\mathrm{T}}$ value of each target gene was calculated by subtraction of the average $\Delta C_{T}$ from the control group. Finally, the $\mathrm{n}$-fold difference was calculated using the formula $2^{-\triangle \Delta C T}$. We used following primers for QPCR: Bax (forward 5'-GGGGATTTCTGACTTGAGG-3' reverse 5'-TTGGGCTGATTTGATTTCTG-3'), Bcl-2 (forward 5'-CGACTTCGCCGAGATGTCCAGCCAG-3' reverse 5'-ACTTGTGGCCCAGATAGGCACCCAG-3').

The cells cultured under standard conditions and without lectin treatment served as a control in our experiment.

Our data are presented as the mean with the standard error of the mean (SEM). The comparison between experiment variants was performed with twoailed Student $t$-test using Microsoft Excel software.

\section{RESULTS AND DISCUSSION}

In our previous study we showed that PHA-L, PHA-E and PHA inhibited the growth of human cancer cells in vitro at concentrations from 1 to $1000 \mu \mathrm{g} / \mathrm{ml}$ [8]. We hypothesized that studied proteins have cytotoxic activity and/or are able to induce apoptosis. Therefore in this study, we have focused on the analysis of apoptotic action of PHA lectins on tumor cells in culture.

As a result of experiment, we have revealed that PHA and its isolectins cause the increase of the percentage of apoptotic cells. We have shown the increase of early apoptotic cells after treatment by all studied lectins at $1 \mu \mathrm{g} / \mathrm{ml}$ compared to control (Fig. 1, a). The highest percentage of dead and apoptotic cells has been observed after treatment with
PHA-E. At the same time, treatment with PHA-L did not significantly influence the dead cell number. It is interesting that percentage of apoptotic cells after PHA and PHA-L treatment was more than twice higher than in control (Fig. 1, a). In our experiment we observed significant reduction of alive cells after treatment with higher concentrations of studied lectins -10 and $100 \mu \mathrm{g} / \mathrm{ml}$ (Fig. 1, b, c). At the same time, the number of apoptotic and dead cells was increased (Fig. 1, $b, c$ ). We would like to note that after the treatment with $\mathrm{PHA}$ and PHA-L at concentrations of 10 and $100 \mu \mathrm{g} / \mathrm{ml}$, higher percentage of apoptotic cells than dead cells has been observed. Meanwhile, after the treatment with PHA-E at these concentrations we registered only dead cells without apoptotic cells (Fig. 1, $b, c$ ).
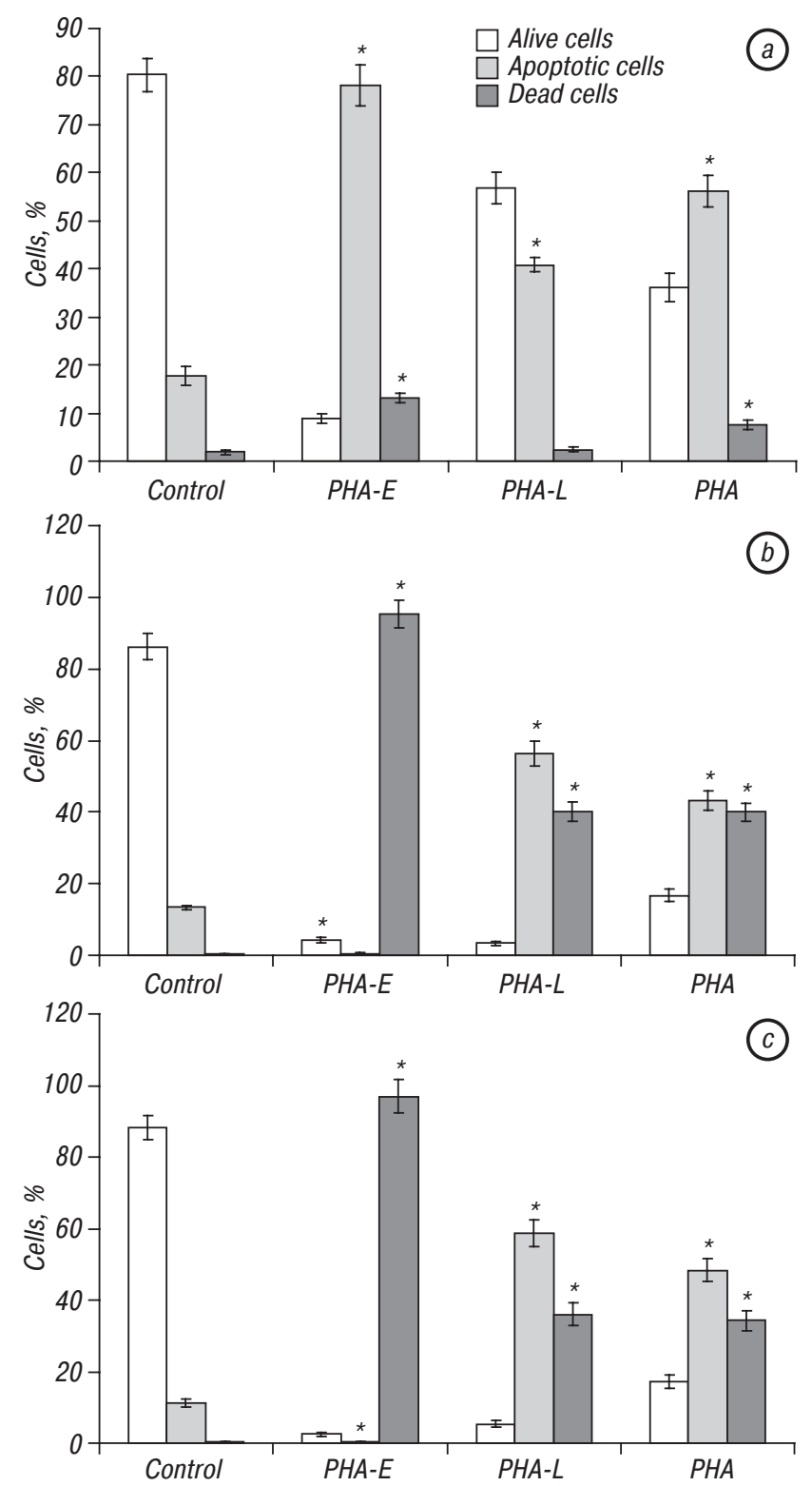

Fig. 1. Cell death and apoptosis in HEp-2 cells induced by PHA and its isolectins at concentrations of $1 \mu \mathrm{g} / \mathrm{ml}(a), 10 \mu \mathrm{g} / \mathrm{ml}(b)$, $100 \mu \mathrm{g} / \mathrm{ml}(c) .{ }^{*} \mathrm{p}<0.05$ compared to control $(\mathrm{n}=3)$

There were a high percentage of cells with apoptotic features under the treatment with all studied lectins at concentration of $1 \mu \mathrm{g} / \mathrm{ml}$. Subsequently, the study of the molecular mechanisms of apoptosis was conducted under the following conditions. 
At the next stage of our work we focused on molecular pathways of PHA lectins activity. We revealed increased level of activated caspase-3 and proapoptotic protein Bax in HEp-2 cells after treatment with PHA-E using western blotting. The level of activated caspase-3 and Bax was 4 and 2.5 fold higher, compared to the untreated cells, respectively (Fig. 2, $a-c$ ). It should be noted that PHA-E increased the level of activated caspase- 3 in cells two times higher than PHA-L or PHA (Fig. 2, a-c). Moreover, we observed higher rate of apoptotic and dead cells and higher level of activated caspase-3 after PHA-Etreatment in our experiment. This increase was statistically significant, compared to the control. Our observation suggests stronger proapoptotic effect of PHA-E comparing to $\mathrm{PHA}$ and $\mathrm{PHA}-\mathrm{L}$.

Western-blot analysis revealed that all investigated lectins increase the level of Bax protein in human HEp-2 carcinoma cells more than 2.5 fold (Fig. 2, a, c). Interestingly, that after PHA-L treatment we observed lower percentage of apoptotic cells and level of the Bax protein, compared to PHA-E or PHA treatment (Fig. 2, a, c).

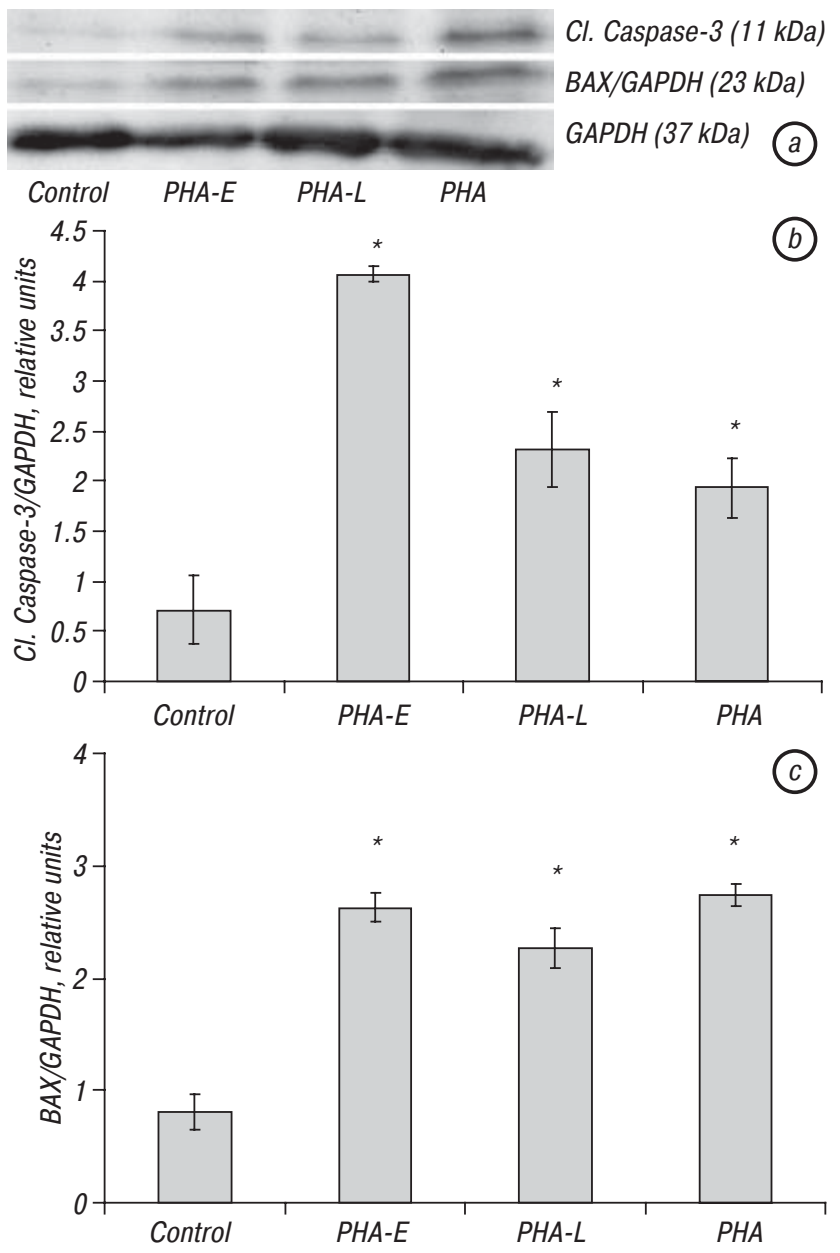

Fig. 2. Expression of cleaved form of caspase-3 (P11) and Bax protein in HEp-2 cells after lectins treatment: $a$ - western blot analysis of HEp-2 cell lysates; $b$ - cleaved caspase-3 level normalized by GAPDH intensity signal; $c-$ Bax level normalized by GAPDH intensity signal. ${ }^{*} p<0.05$ compared to control $(n=3)$

Using qPCR we observed that all analyzed PHA lectins up-regulated level of Bax gene expression, compared to control. Higher level of Bax gene expression was observed after PHA-E treatment (Fig. 3). However, PHA-L and PHA also increased the Bax gene expres- sion more than 2 times. These findings are in agreement with the data of western blot mentioned above. Thus, our data indicate the ability of all studied PHA lectins to induce apoptosis in human HEp-2 carcinoma cells cultures through caspase 3 and Bax activation.

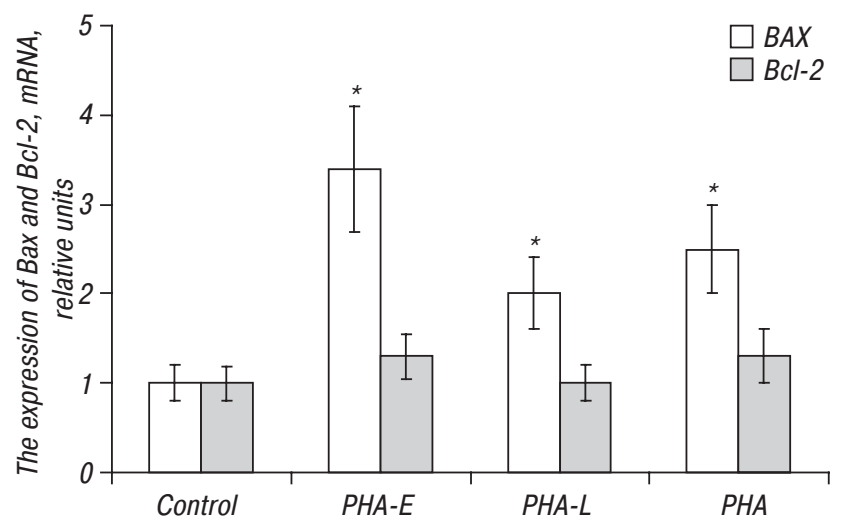

Fig. 3. Expression of $B a x$ and $B C l-2$ mRNAs in HEp-2 cells after lectins treatment. ${ }^{*} p<0.05$ compared to control $(n=3)$

In our experiment we also have analyzed the level of $\mathrm{Bcl}-2$ gene expression in treated and control cells. Using qPCR we showed that all analyzed lectins did not affect the level of $\mathrm{Bcl}-2$ gene expression in $\mathrm{HEp}-2$ cells (see Fig. 3). Bcl-2 was expressed at the same level in control and after the treatment of HEp-2 cells with $\mathrm{PHA}$ and its isolectins. Bcl-2 protein has an antiapoptotic activity and is known as the extremely important regulator of apoptosis [15].

Thus, we have shown that PHA and its isolectins are able to induce apoptosis in the human tumor cells. We revealed that all studied lectins activated the effector caspases-3 and the proapoptotic Bax expression on the protein and RNA levels. At the same time, we did not observe any changes of antiapoptotic $\mathrm{Bcl}-2$ gene expression after PHA and its isolectins treatment of tumor cells. It is important to note that in our experiment PHA-E induced apoptotic cell death more effectively than other studied proteins. We have observed the higher percentage of apoptotic cells in culture, higher level of activated caspase-3 and Bax gene in case of PHA-E treatment. We suppose that the discrepancy in the activity of studied lectins could be explained by their carbohydrate specificity.

We would like to note that our data are in agreement with previously published data [8]. Authors reported about apoptosis induction by PHA-E in lung cancer cell culture through mitochondrial signalling activation [7]. In our work we have shown that not only PHA-E can lead to apoptosis induction but other PHA lectins also. In literature, specific carbohydrate recognising and carbohydrate binding properties of lectins play principle role in cell-lectin interaction and recognition of cancer cells by lectins [1]. Based on our and literature data, we assume that PHA-E is able to induce apoptosis via different signalling mechanisms not only in cancer cells.

Thus, our data indicate that total PHA and its isolectins are able to induce apoptosis in human tumor cells through up-regulation of Bax protein and activation of the effector caspase-3. 


\section{REFERENCES}

1. Antonyuk VO. Lectins and theirs sources of raw materials. Lviv: Kvart, 2005. 554 p. (in Russian).

2. Chunhua R, Zhao RC. Essentials of mesenchymal stem cell biology and its clinical translation. Dordrecht: Springer Science \& Business Media, 2013. 321 p.

3. Gatehouse AMR, Dewey FM, Dove I, et al. Effect of seed lectins from Phaseolus vulgaris on development of larvae of Callosobruchus maculatus: mechanism of toxicity. J Sci Food Agr 1984; 35: 373-80.

4. Bardocz S, Grant G, Pusztai A. The effect of phytohaemagglutinin at different dietary concentration on the growth, body composition and plasma insulin of the rat. $\mathrm{Br} \mathrm{J}$ Nutr 1996; 76: 613-26.

5. Baintner K, Kiss P, Bardocz S, et al. Origin and mediation of secretion induced by oral phytohaemagglutinin (PHA) in rats. Acta Physiol Hung 2004; 91: 221-33.

6. Kumar S, Verma AK, Sharma A, et al. Phytohemagglutinins augment red kidney bean (Phaseolus vulgaris L.) induced allergic manifestations. J Proteomics 2013; 93: 50-64.

7. Kuo WT, Ho YJ, Kuo SM, et al. Induction of the mitochondria apoptosis pathway by phytohemagglutinin erythroagglutinating in human lung cancer cells. Ann Surg Oncol 2011; 18: 848-56.

8. Kochubei TO, Piven OO, Andrienko VI, et al. Influence of Phaseolus vulgaris phytohemagglutinin and its isoforms on the proliferation and survival of mammalian cells in vitro. Visn Ukr Tov Genet Sel 2012; 10: 42-50 (in Ukrainian).

9. Yamamoto H, Swoger J, Greene S, et al. Beta1,6-Nacetylglucosamine-bearing $\mathrm{N}$-glycans in human gliomas: implications for a role in regulating invasivity. Cancer Res 2000; 60: $134-42$.

10. Lukash LL, Paton EB, Sukhorada EM, et al. The evaluation of the cytotoxicity of preparations with anticarcinogenic action in human cell cultures. Tsitol Genet 1997; 31: 26-34 (in Russian).

11. McGanon AJ, Martin SJ, Bissonnette RP, et al. The end of the (cell) line: methods for the study of apoptosis in vitro. Methods Cell Biol 1995; 46: 153-85.

12. Maksymchuk OV, Bezdrobna LK, Sidorik LL, et al. Cytochrome P450 2E1 expression in mice liver under exposure of continuous and acute $\gamma$-radiation. Ukr Biokhim Zhurn 2008; 80: 59-65 (in Ukrainian).

13. Morton EN, Margison GP. Increased $\mathrm{O}^{6}$-alkylguanine-DNA alkyltransferase activity in Chinese hamster V-79 cells following selection with chloroethylating agents. Carcinogenesis 1988; 9: 45-9.

14. Laemmli UK. Cleavage of structural proteins during the assembly of the head of bacteriophage T4. Nature 1970; 227: $680-5$.

15. Elmore S. Apoptosis: a rewiew of programmed cell death. Toxicol Pathol 2007; 35: 495-516. 